\title{
Is the presence of left atrial diverticulum associated with recurrence in patients undergoing catheter ablation for atrial fibrillation?
}

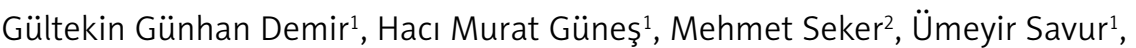 \\ Gamze Babur Güler ${ }^{1}$, Ekrem Güler ${ }^{1}$, Mehmet Dogan ${ }^{1}$, Cengiz Erol' ${ }^{2}$, Fethi Kılıçaslan ${ }^{1}$
}

${ }^{1}$ Department of Cardiology, Istanbul Medipol University, Istanbul, Turkey
2Department of Radiology, Istanbul Medipol University, Istanbul, Turkey

Submitted: 11 January 2019

Accepted: 10 February 2019

Arch Med Sci Atheroscler Dis 2019; 4: e25-e31

DOI: https://doi.org/10.5114/amsad.2019.83508

Copyright (c) 2019 Termedia \& Banach

\section{Abstract}

Introduction: Catheter ablation (CA) for atrial fibrillation (AF) has been a major cornerstone in the management of AF. Despite promising advances in CA techniques, long-term results reveal a high rate of recurrence after the procedure. Left atrial diverticulum (LAD), a common anatomic variant of the left atrium, was previously shown to be associated with increased risk of thrombus formation, cardiac perforation and arrhythmia. In this study we aimed to investigate the relationship between LAD and recurrence in patients undergoing CA for AF.

Material and methods: A total of 100 consecutive patients with a mean age of $53 \pm 12.1$ years ( $53 \%$ male) underwent radiofrequency (RF) $(46,46 \%$ ) or cryoballoon $(54,54 \%)$ catheter ablation for atrial fibrillation preceded by cardiac computed tomography (CT) imaging. Clinical and procedural characteristics of the patients with and without AF recurrence were compared. Results: Twenty-three (23\%) patients had AF recurrence and 77 (77\%) patients had no recurrence. The clinical parameters such as hypertension, diabetes mellitus, coronary artery disease and stroke did not differ between the groups. Left atrium diameter was significantly different between the two groups ( $4.1 \pm 0.5$ vs. $3.9 \pm 0.5, p=0.042)$. Presence of LAD was not different between the two groups (7 (31.8) vs. 21 (28.8); $p=0.794)$. Multivariate logistic regression analysis revealed RF ablation as the most important independent variable for AF recurrence $(\beta=3.115, p<0.001, \mathrm{OR}=22.526$, $95 \% \mathrm{Cl}: 4.287-118.351)$.

Conclusions: The presence of left atrial diverticulum is not associated with recurrence in patients undergoing RF and cryoballoon CA for atrial fibrillation.

Key words: atrial fibrillation, catheter ablation, left atrial diverticulum.

\section{Introduction}

Catheter ablation (CA) for atrial fibrillation (AF), the most common sustained arrhythmia, represents an increasingly used option in the armamentarium of AF management. However, when compared with acute success rates of CA for supraventricular tachyarrhythmias other than AF, it still has a remarkably lower acute success rate in spite of technologic advances in catheter design and imaging quality. Similarly, in the long term a significant number of patients (30-50\%) undergoing CA develop
Corresponding author:

Dr. Gültekin Günhan Demir Department of Cardiology Istanbul Medipol University

Istanbul, Turkey

Phone: +905336320488

E-mail: ggdemir@gmail.com 
recurrence [1]. Although multiple risk factors for AF recurrence have been identified, weak predictive power has prevented their integrated use in daily practice.

Left atrial diverticulum (LAD) is a common anatomical variant of the left atrium with a wide range of prevalence of $10-46 \%$ in recent reports [2]. The presence of LAD has recently been shown to be associated with increased risk for thrombus formation, cardiac perforation and arrhythmia [3]. However, the relationship between the presence of $L A D$ and recurrence after radiofrequency and cryoballoon CA for atrial fibrillation has not been evaluated so far.

\section{Aim}

The purpose of the present study was to investigate the relationship between LAD and recurrence undergoing CA for atrial fibrillation.

\section{Material and methods}

\section{Patient population}

In this study with a retrospective design, we enrolled a total of 100 patients with paroxysmal or persistent AF who were refractory/intolerant to antiarrhythmic drug therapy and underwent $A F$ ablation with radiofrequency (RF) or cryoballoon CA in Istanbul Medipol University between January 2012 and December 2016. Paroxysmal AF was defined as a self-terminating AF episode within 7 days, and persistent AF was defined as the presence of an AF episode lasting longer than 7 days. Before the ablation, transthoracic and transesophageal echocardiograms were recorded for each patient. Standard echocardiographic measurements were calculated. All of the patients underwent transesophageal echocardiographic examination to rule out intracavitary thrombus. All antiarrhythmic drugs were discontinued for at least 5 half-lives before the ablation procedure. Exclusion criteria included patients with a history of cardiac valve surgery, permanent $A F$, severe valvular heart disease, known allergy to iodine-based contrast medium, patients with documented thrombus in the left atrium or atrial appendage, and patients with poor image quality obtained by echocardiography or computed tomography examination.

The study was approved by Istanbul Medipol University Local Ethical Committee.

\section{CT scan, image interpretation}

All images were obtained with 256-slice CT (Brilliance-iCT; Philips Healthcare, Cleveland, $\mathrm{OH}$, USA) following intravenous contrast material injection $(100 \mathrm{ml} 3 \mathrm{ml} / \mathrm{s}, 128 \mathrm{~mm} \times 0.625 \mathrm{~mm}$ collimation). Electrocardiographic (ECG) gating was performed with a triggered delay set at $70 \%$ of the RR interval. Rate control in patients with high heart rate was achieved with preprocedural intravenous $\beta$-blocker administration. All images were transferred to a workstation for interpretation.

The CT data sets were evaluated using standard axial, sagittal, and coronal reconstructions with additional interactive multiplanar reconstructions, maximum intensity projections, and interactive three-dimensional volume rendering (Figure 1). The images were assessed and reviewed by two radiologists experienced in cardiac CT imaging interpretation (M.Ş, C.E).

A left atrial diverticulum was defined as a protrusion from the heart cavity to outside the left atrial wall plane with a wide neck and a smooth contour to its body [4]. On the basis of the atrial wall, locations of LADs were classified as superior, inferior, right and left lateral, anterior and posterior, and septal. All of the shapes consistent with $L A D$ definition were measured at the major diameter of the LAD orifice and the depth of the LAD. Left atrial accessory appendages were defined as outpouchings with a discernible ostium with a narrow neck and irregular contours suggestive of pectinate muscles.

\section{Ablation procedure}

The cryoballoon ablation technique used during the procedure was similar to those described elsewhere $[5,6]$. Seldinger technique was used for both right and left femoral vein punctures in patients allocated for cryoablation. A 6 French (Fr) decapolar catheter was advanced through the femoral vein into the coronary sinus (CS). Through the right femoral vein a $7 \mathrm{Fr}$ long-sheath was advanced to the superior vena cava guided with a 0.38 inch guidewire. A Brockenbrough needle (St. Jude Medical) was used for transseptal puncture guided by fluoroscopy. In patients considered difficult for transseptal puncture, transesophageal echocardiography was used. A steerable $12 \mathrm{Fr}$ sheath (FlexCath, Medtronic) was advanced to the left atrium after successful puncture. For cryoablation, a 28-mm cryoballoon (Arctic Front Medtronic Cryocath and Arctic Front Advance) was used. An Achieve guidewire (Medtronic Ablation Frontiers, LLC, Carlsbad, CA) was used to direct the balloon into the PV ostium for mapping PV potentials before, during and after cryoablation applications. For visual confirmation of balloon occlusion, contrast medium was injected into the distal area of the cryoballoon through the Arctic Front catheter. Two cryoapplications were performed for each PV and each application was delivered for $4 \mathrm{~min}$. In cases of persistence of PV potentials, one extra cryoapplication was attempted. In order to avoid phrenic nerve palsy, the decapolar CS catheter 

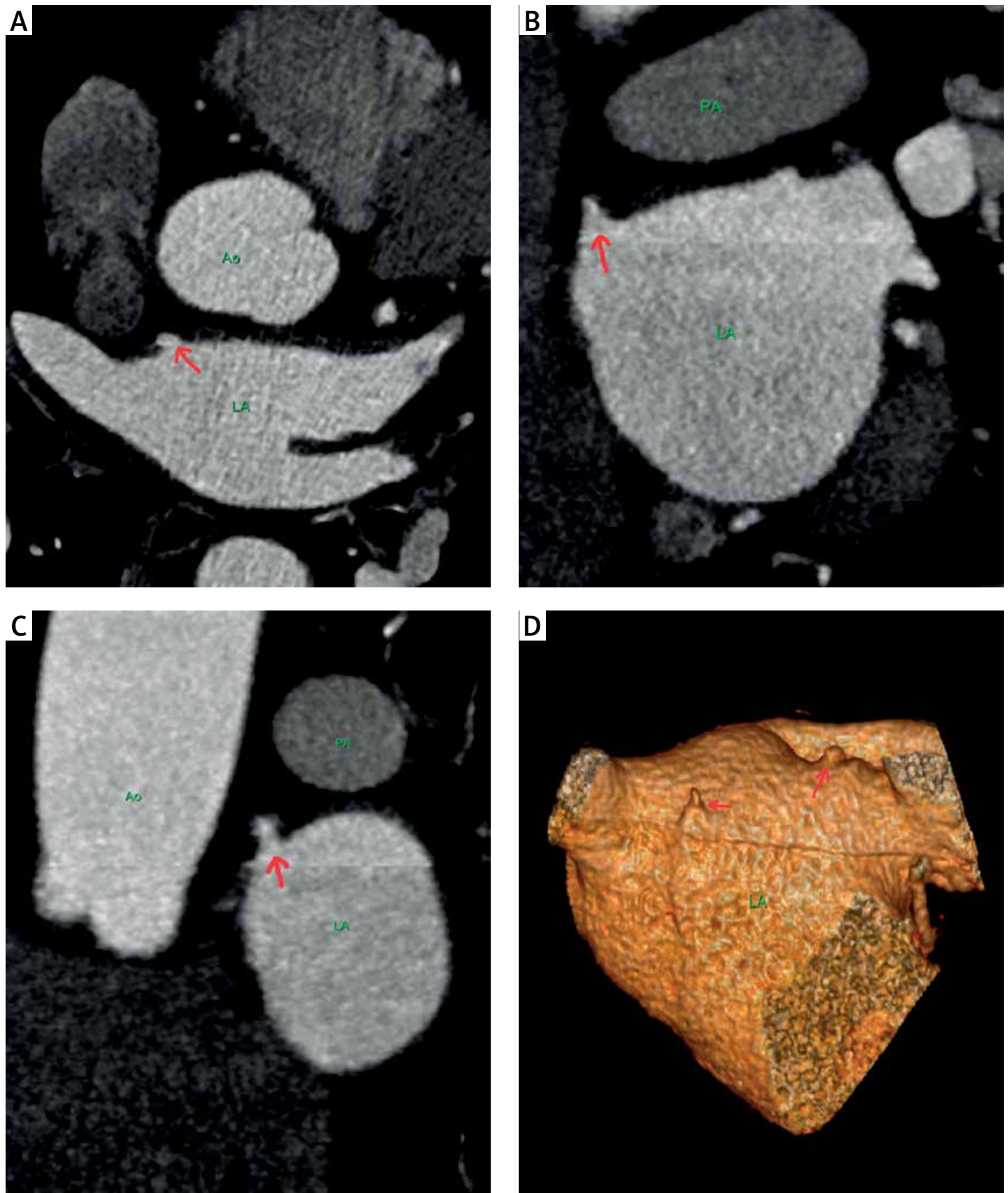

Figure 1. 50-year-old man with left atrial diverticula. Axial (A), coronal (B), sagittal (C), and 3D reconstruction (D) images show left atrium with a small diverticula (red arrow) located in the right anterosuperior wall. Note 3D reconstruction image shows another small diverticula (red arrows) located in the left anterosuperior wall close to the ostia of the right superior pulmonary vein

$L A$ - left atrium, Ao - aorta, PA - pulmonary artery.

was placed in the superior vena cava for continuous phrenic nerve pacing during ablation before targeting the right PVs.

The radiofrequency ablation technique used during the procedure was similar to those described elsewhere [7, 8]. Femoral access was achieved by two right and one left femoral vein punctures with Seldinger technique in patients undergoing RF ablation. A 6 Fr decapolar catheter was advanced into the CS via the left femoral vein. A Brockenbrough needle (St. Jude Medical) was used for transseptal puncture guided by fluoroscopy. In patients considered difficult for transseptal puncture, transesophageal echocardiography was used. Following transseptal puncture two catheters, a circumferential PV mapping catheter (Lasso TM, Biosense and Webster, Inc., CA, USA) and a 4-mm cooled-tip ablation catheter (ThermoCool Navi-Star, Biosense-Webster, Inc., CA, USA or Medtronic SPRINKLR), were advanced into the 
left atrium. We used an electro-anatomic mapping system (NavX; St. Jude Medical, St. Paul, MN, USA and CARTO; Biosense Webster, Diamond Bar, CA, USA) to guide the ablation procedure. The circular mapping catheter was positioned close to the PV ostium, and point-by-point RF ablation was performed to isolate the right and left PVs. RF energy was delivered in a power-controlled mode with a power limit of $35 \mathrm{~W}$ ( $30 \mathrm{~W}$ at the posterior wall) and a maximal temperature of $45^{\circ} \mathrm{C}$. A voltage of $<0.1 \mathrm{mV}$ was targeted at each point and RF energy was delivered until it was achieved with a maximum of $15 \mathrm{~s}$ per point. Confirmation of PV isolation was made by using entrance and exit blocks. Reconfirmation of PV isolation was performed 20 min after RF ablation for each PV.

For both procedures, bolus heparin $(100 \mathrm{U} / \mathrm{kg})$ was initiated as soon as transseptal puncture was achieved. During the procedure, the activated clotting time was monitored every $30 \mathrm{~min}$, and additional heparin boluses were given to maintain activation clotting time between 300 and $350 \mathrm{~s}$.

\section{Patient follow-up}

All of the patients were provided at least $48 \mathrm{~h}$ of continuous in-hospital ECG monitoring after CA. Patients were scheduled for follow-up visits at 1, 3, 6, 9 and 12 months after CA. Recurrence of AF was defined as the presence of any episode of atrial tachycardia or AF lasting $30 \mathrm{~s}$ or more on 12-lead electrocardiogram (ECG) and/or 24-h ambulatory ECG monitoring at each visit after CA for AF. A blanking period of 3 months was established after RF ablation and cryoablation. The follow-up period was limited to 12 months in order to detect recurrences; very late recurrences (after $>12$ months) were excluded. A repeat procedure was offered to patients presenting with recurrence $>3$ months after the index procedure.

\section{Statistical analysis}

Mean \pm standard deviation and median (minimum and maximum values) were used for continuous variables, whereas categorical variables were presented as number of cases (percentage). Normal distribution was tested with the Kolmogorov-Smirnov test and was confirmed with skewness and kurtosis. Comparison between the groups with and without AF recurrence was made by the means of unpaired Student's $t$-test for continuous variables and the Mann-Whitney test was used for non-normally distributed continuous data. Fisher's exact and continuity correction (Yates' correction) tests were used to compare categorical variables. In order to predict AF recurrence the variables which were significant at the $10 \%$ level in univariate statistics were further evaluated with age and LAD frequency adjusted multivariate logistic regression analysis (enter model). The results of the model were reported as the odd ratio (OR), 95\% confidence interval, $\beta$ and $p$-values. SPSS version 11.0 (SPSS Inc., Chicago, IL, USA) was used for statistical analysis. $P<0.05$ was considered significant for all analyses.

\section{Results}

One hundred consecutive patients with a mean age of $53 \pm 12.1$ years ( $53 \%$ male) underwent CA for AF preceded by cardiac CT imaging. Comparative baseline clinical and procedural characteristics of patients with and without recurrence are provided in Table I. Twenty-three (23\%) AF patients had $A F$ recurrence and 77 (77\%) patients had no recurrence. The clinical parameters such as hypertension, diabetes mellitus, coronary artery disease and stroke did not differ between the groups. Left atrium diameter was significantly different between the two groups ( $4.1 \pm 0.5$ vs. $3.9 \pm 0.5, p=$ 0.042) (Table I). Presence of left atrial diverticulum was not different between the two groups $(n=7$ (31.8) vs. 21 (28.8); $p=0.794)$. Twenty (87\%) patients in the RF ablation group had recurrence of AF while only 3 (13\%) patients treated with cryoballoon CA had recurrence.

We present a multivariate logistic regression analysis to determine the independent predictors of AF recurrence, in particular the presence of LAD, in Table II. In the logistic regression model, we used the significant variables $(p<0.10)$ such as $L A D$, persistent $A F, R F$ ablation procedure from univariate analyses with age and presence of diverticulum. Among these, RF ablation procedure was found as the most important independent variable of AF recurrence $(\beta=3.115, p<0.001$, $\mathrm{OR}=22.526,95 \% \mathrm{Cl}: 4.287-118.351$ ) (Table II).

\section{Discussion}

The main finding of this study is that the presence of LAD is not associated with AF recurrence in patients undergoing RF and cryoballoon CA for atrial fibrillation. Left atrial diverticulum is assumed to contract in synchrony with the rest of the atrium as it consists of normal myocardial wall structure [9]. Left atrial diverticula are thought to represent remnants of the cardinal venous system (stump or failure of regression) during embryologic development [9]. The prevalence of left atrial diverticulum has been reported to vary between 10 and $46 \%$ in the literature [2, 3, 10]. Hołda et al. reported the presence of LAD as $18 \%$ in their study, which examined heart anatomy in 200 autopsied, non-atrial fibrillation hearts [11]. Previous studies did not detect a significant difference in the prevalence of $L A D$ between patients in sinus 
Table I. Comparison of clinical and procedural characteristics of patients with and without recurrence

\begin{tabular}{|lccc|}
\hline Parameter & Recurrence (+) & Recurrence (-) & $P$-value \\
\hline Sex (male), $n$ (\%) & $10(43.5)$ & $43(55.8)$ & 0.421 \\
\hline Age [years] & $53 \pm 13$ & $54 \pm 12$ & 0.585 \\
\hline EF(\%) (range) & $65(66-45)$ & $65(70-35)$ & 0.159 \\
\hline EDD [cm] & $4.76 \pm 0.37$ & $4.79 \pm 0.40$ & 0.807 \\
\hline Persistent AF, $n$ (\%) & $3(13)$ & $2(2.6)$ & 0.078 \\
\hline HT, $n(\%)$ & $9(39.1)$ & $44(57.1)$ & 0.157 \\
\hline DM, $n$ (\%) & $11(14.3)$ & $2(8.7)$ & 0.727 \\
\hline CAD, $n(\%)$ & $6(26.1)$ & $23(29.9)$ & 0.799 \\
\hline Stroke, $n$ (\%) & $1(4.3)$ & $2(2.6)$ & 0.548 \\
\hline LA diameter & $4.1 \pm 0.5$ & $3.9 \pm 0.5$ & 0.042 \\
\hline Diverticulum, $n$ (\%) & $7(31.8)$ & $21(28.8)$ & $<.794$ \\
\hline Procedure type, $n$ (\%): & & $26(33.8)$ & \\
\hline \begin{tabular}{l} 
RFA \\
\hline Cryoballoon CA
\end{tabular} & $20(87)$ & $51(66.2)$ & \\
\hline
\end{tabular}

$A F$ - atrial fibrillation, CA - catheter ablation, CAD - coronary artery disease, DM - diabetes mellitus, EDD - end-diastolic diameter, EF- ejection fraction, HT - hypertension, LA - left atrium, RFA - radiofrequency ablation.

Table II. Multivariate logistic regression analysis to determine independent predictors of recurrence of atrial fibrillation after catheter ablation

\begin{tabular}{|lcccc|}
\hline Parameter & $\beta$ & $P$-value & OR & $95 \% \mathrm{Cl}$ \\
\hline LA diameter & 0.896 & 0.210 & 2.449 & $0.604-9.922$ \\
\hline Persistent AF & 1.321 & 0.276 & 3.748 & $0.348-40.356$ \\
\hline RFA & 3.115 & $<0.001$ & 22.526 & $4.287-118.351$ \\
\hline Diverticulum & 0.705 & 0.291 & 2.024 & $0.547-7.490$ \\
\hline Age & -0.057 & 0.077 & 0.945 & $0.887-1.006$ \\
\hline
\end{tabular}

$A F$ - atrial fibrillation, LA - left atrium, RFA - radiofrequency ablation.

rhythm and atrial fibrillation [2, 12, 13]. DePonti et al. investigated the morphology and clinical impact of LAD in 212 patients with AF undergoing RF ablation and found an LAD prevalence of $25 \%$ in their study group. We had a similar rate of patients with LAD (28\%) in our study population, consistent with the literature.

Previous studies commonly focused on left atrial anatomical abnormalities/variants and their associations with $\mathrm{AF}$ therefore, when compared with LAD left atrial accessory appendages, were more deeply evaluated [9]. Outpouchings from the left atrial wall with a narrow neck and irregular contours are suggestive of an accessory appendage, while LAD has a broad neck and smooth contour[14].

Meanwhile, the abovementioned De Ponti et al. were the only authors who focused on the outcomes of patients with $L A D$ and AF undergoing CA. Similarly, they detected no significant differ- ence in terms of recurrence of $\mathrm{AF}$ between patients with and without LAD. However, our study has 2 important differences from that study: i) the present study included patients undergoing not only RF ablation but also cryoballoon CA; ii) the definition of LAD was used as an umbrella term which included true LAD, accessory appendages and aneurysms in their study, while we included only patients with true LAD.

Although there has been a considerable paucity of articles about $L A D$ in recent years, the main concerns in studies assessing LAD included its potential role in procedure-related complications (i.e. catheter entrapment, cardiac perforation due to thinner wall structure), intracavitary thrombus formation and arrhythmogenic activity [9]. The former two relationships are outside the scope of interest of the present study, but before mentioning the association between LAD and atrial 
arrhythmic activity, one has to keep in mind that a vast majority of the previous studies evaluating this topic were conducted by radiologists and published in radiology journals. The papers used as references for identification of ectopic focal electrical activity in LAD/left atrial wall accessory appendages are limited to exceptionally rare case reports $[2,9,15]$. However, when compared with left atrium accessory appendages, less is known about the ectopic activity and electrophysiological characteristics of LAD. The main suggested mechanism was the contiguity of the LAD and myocardial wall/sleeve and the potential for giving rise to arrhythmogenic activity. Despite being theoretically reasonable, none of the contemporary reports found a causative relationship between LAD and arrhythmogenic activity except several case reports [16]. Therefore, the lack of association between recurrence of $A F$ and $L A D$ in our study is supported by previous reports.

The exact mechanism underlying AF recurrence is ill defined and the proposed mechanisms in the literature mainly include technical failure, disease progression, atrial structural remodeling, individual patient characteristics and comorbidities [17]. A true LAD is composed of normal myocardial wall array and is supposed to show synchronized contraction with the rest of the atrium.

The only study focusing on basic electrophysiological characteristics (impedance, voltage, electrograms) of LAD was conducted by Tan et al., who demonstrated a significant difference between areas without LAD [18]. However, the study population consisted of only 24 patients and the main objective was use of electrophysiological characteristics to help differentiation of LAD (electroanatomical mapping) during $C A$ for $A F$ in order to avoid procedural complications rather than prediction of recurrence. Furthermore, ectopic activity in LAD or its association with recurrence of AF was not targeted, which differs from our study.

Another finding in the present study revealed by multivariate logistic regression analysis was detection of RF ablation as a strong predictor for recurrence of $A F$. RF ablation for $A F$ is the preferred ablation method for patients with more complex anatomy in daily interventional practice, so when this is taken into account, it is not a surprise to see patients with more complex anatomy and arrhythmia suffering more episodes of recurrence. Although we did not intend to compare the efficacy and safety of the two ablation methods irrespective of the presence of $L A D$, the limited number of patients in our study was probably the primary factor responsible for this discrepancy.

Presence of LAD may be associated with lower success rates and increased procedural complications such as perforation and coronary artery injury. In our study population 2 patients treated with cryoballoon CA experienced complications (1 phrenic nerve palsy and 1 femoral arteriovenous fistula) and 2 patients treated with RF CA suffered complications (1 pulmonary vein stenosis, 1 femoral hematoma). Furthermore, all of the complications developed in patients without LAD.

In an exploratory study, Patel et al. investigated the prevalence of LAD and accessory appendage and their association with adverse outcomes in 46 patients undergoing RF ablation for $\mathrm{AF}$ and found that presence of LAD or left atrial accessory appendage was unrelated to adverse outcomes [13]. Although this study was regarded as exploratory, it has similar findings with our study such as lack of association with LAD and recurrence of $A F$ and a similar rate of LAD prevalence $(30 \%)$ in patients undergoing CA. However, unlike our study they did not evaluate patients undergoing cryoballoon CA and the representation of LAD under the term 'normal variation left atrial anatomy', which also included accessory appendages, was weak.

The majority of previous studies investigating the relationship between $L A D$ prevalence and CA addressed $L A D$ and accessory appendages together as normal variation of left atrial anatomy while we specifically focused on the prevalence of LAD and clinical outcomes. Another strength of our study is the investigation of the relationship between LAD and recurrence of AF after cryoballoon CA for the first time. The main limitation of our study was the relatively small sample size and retrospective design. Randomization of patients to cryoballoon or RF CA was left to the discretion of the operator; therefore the possibility of selection bias cannot be excluded. Similarly, detection of RF CA as a strong predictor of AF recurrence in our multivariate analysis may be associated with preference of RF CA in patients with more complex anatomy, longer duration of AF, larger LA diameter and comorbidities. The quality of follow-up can be considered as a limitation. Some studies used 5or 7-day Holter-ECG monitoring, but most of the studies in the literature used 24-h monitoring. The association between the locations of LADs and recurrence could be investigated perhaps in a larger sample size. Performance of all ablation procedures in a single center may be considered as another limitation. Electrophysiological data regarding electrical activity within the LAD could have provided more accurate information about the pathophysiology of recurrence.

In conclusion, the presence of left atrial diverticulum is not associated with recurrence in patients undergoing RF and cryoballoon CA for atrial fibrillation.

\section{Conflict of interest}

The authors declare no conflict of interest. 


\section{References}

1. Kirchhof P, Benussi S, Kotecha D, et al. 2016 ESC Guidelines for the management of atrial fibrillation developed in collaboration with EACTS. Europace 2016; 18: 1609-78.

2. Troupis J, Crossett M, Scneider-Kolsky M, Nandurkar D. Presence of accessory left atrial appendage/diverticula in a population with atrial fibrillation compared with those in sinus rhythm: a retrospective review. Int J Cardiovasc Imaging 2012; 28: 375-80.

3. De Ponti R, Lumia D, Marazzi R, et al. Left atrial diverticula in patients undergoing atrial fibrillation ablation: morphologic analysis and clinical impact. J Cardiovasc Electrophysiol 2013; 24: 1232-9.

4. Chen J, Yang ZG, Xu HY, et al. Assessments of pulmonary vein and left atrial anatomical variants in atrial fibrillation patients for catheter ablation with cardiac CT. Eur Radiol 2017; 27: 660-70.

5. Neumann T, Vogt J, Schumacher B, et al. Circumferential pulmonary vein isolation with the cryoballoon technique results from a prospective 3-center study. J Am Coll Cardiol 2008; 52: 273-8.

6. Van Belle $Y$, Janse P, Rivero-Ayerza MJ, et al. Pulmonary vein isolation using an occluding cryoballoon for circumferential ablation: feasibility, complications, and short-term outcome. Eur Heart J 2007; 28: 2231-7.

7. Deisenhofer I, Schmitt C. Ablation strategies in paroxysmal atrial fibrillation. In: Catheter Ablation of Atrial Fibrillation. Aliot E, Haissaguerre M, Jackman WM (eds.). Blackwell 2008; 136-62.

8. Ernst S, Ouyang F, Antz M, et al. Catheter ablation of atrial fibrillation. In: Techniques Targeting the Pulmonary Veins. Aliot E, Haissaguerre M, Jackman WM (eds.). Blackwell 2008; 117-23.

9. Lazoura O, Reddy T, Shriharan M, et al. Prevalence of left atrial anatomical abnormalities in patients with recurrent atrial fibrillation compared with patients in sinus rhythm using multi-slice CT. J Cardiovasc Comput Tomogr 2012; 6: 268-73.

10. Incedayi M, Öztürk E, Sonmez G, et al. The incidence of left atrial diverticula in coronary CT angiography. Diagn Interv Radiol 2012; 18: 542-6.

11. Hołda MK, Koziej M, Hołda J, et al. Anatomic characteristics of the mitral isthmus region: the left atrial appendage isthmus as a possible ablation target. Ann Anat 2017; 210: 103-11.

12. Terpenning S, Ketai LH, Teague SD, Rissing SM. Prevalence of left atrial abnormalities in atrial fibrillation versus normal sinus patients. Acta Radiol Open 2016; 5: 2058460116651899.

13. Patel SN, French A, Mathias H, Lyen S, Hamilton MC, Manghat NE. Presence of left atrial diverticula, accessory appendages, and normal variant pulmonary venous anatomy diagnosed using MDCT and adverse outcomes following radiofrequency catheter ablation therapy in patients with drug-refractory atrial fibrillation: an exploratory study. Clin Radiol 2013; 68: 762-9.

14. Abbara S, Mundo-Sagardia JA, Hoffmann U, Cury RC. Cardiac CT assessment of left atrial accessory appendages and diverticula. AJR Am J Roentgenol 2009; 193: 807-12.

15. Srinivasan V, Levinsky L, Idbeis B, et al. Congenital diverticulum of the left atrium. Cardiovasc Dis 1980; 7: 405-10.

16. Peng LQ, Yu JQ, Yang ZG, et al. Left atrial diverticula in patients referred for radiofrequency ablation of atrial fibrillation: assessment of prevalence and morphologic characteristics by dual-source computed tomography. Circ Arrhythm Electrophysiol 2012; 5: 345-50.
17. Yang PS, Park YA, Kim TH, et al. Which patients recur as atrial tachycardia rather than atrial fibrillation after catheter ablation of atrial fibrillation? PLoS One 2017; 12: e0188326.

18. Tan C, Han W, Liu X, et al. Electrophysiological characteristics of left atrial diverticulum in patients with atrial fibrillation: electrograms, impedance and clinical implications. Int J Cardiol 2014; 176: 48-54. 